\title{
La téléconduite des usines du Rhin
}

\author{
Philippe Laurençon
}

E.D.F.-G.R.P.H. Rhin

\section{L'aménagement du Rhin}

Sur les $183 \mathrm{~km}$ de son parcours franco-allemand, le Rhin subit une dénivellation de $142 \mathrm{~m}$ pour ụn débit moyen à Bâle de $1060 \mathrm{~m}^{3} / \mathrm{s}$.

En 1919, le traité de Versailles qui marquait la fin de la $1^{\text {re }}$ guerre mondiale, a réservé à la France le droit d'exploiter les ressources énergétiques du fleuve dans le secteur compris entre Bâle et Lauterbourg (cf. fig. l).

L'équipement hydrơelectrique devait en contre-partie aller de pair avec la construction d'écluses pour assurer la continuité de la voie d'eau internationale constituée par le Rhin.

Les 4 premières usines, Kembs, Ottmarsheim, Fessenheim et Vogelgrun ont été construites entre 1932 et 1959 , sur un canal latéral situé en rive gauche. Ce canal, le "Grand Canal d'Alsace », prend son origine au barrage mobile de Kembs implanté dans le lit du fleuve.

Les 4 usines suivantes, Marckolsheim, Rhinau, Gerstheim et Strasbourg ont ensuite été construites entre 1961 et 1970, chacune sur un canal particulier implanté également en rive gauche. A chaque usine correspond alors un barrage mobile effectuant la séparation de chaque canal de dérivation avec le lit du fleuve.

Les 2 dernières usines enfin, Gambsheim et Iffezheim ont été construites en 1974 et 1977 à proximité immédiate du lit naturel, sur le même axe transversal que le barrage mobile correspondant.

La concession et l'exploitation des 8 premiers aménagements (de Kembs à Strasbourg) est attribuée à Electricité de France qui prend également en charge l'exploitation des écluses accolées aux usines et des canaux de dérivation.

Les usines de Gambsheim et d'Iffezheim sont concédées respectivement à CERGA (Centrale électrique rhénane de Gambsheim), société de droit français, et R.K.I. (Rheinkraftwerk Iffezheim), société de droit allemand dans lesquelles E.D.F. et Badenwerk (Société de production et de distribution d'électricité du Land de Bade-Wurtemberg en R.F.A.) ont chacun une participation de $50 \%$. L'exploitation de ces deux centrales est effectuée respectivement par E.D.F. et Badenwerk, tandis que les productions de chaque usine sont partagées entre les deux partenaires. Les écluses et canaux de dérivation de ces deux aménagements sont pris en charge par les services français et allemand de la Navigation.

Cette partie de l'aménagement du Rhin représente (y compris la part de Badenwerk) une puissance totale de près de $1400 \mathrm{MW}$ pour une production annuelle moyenne d'environ $8600 \mathrm{GWh}$.

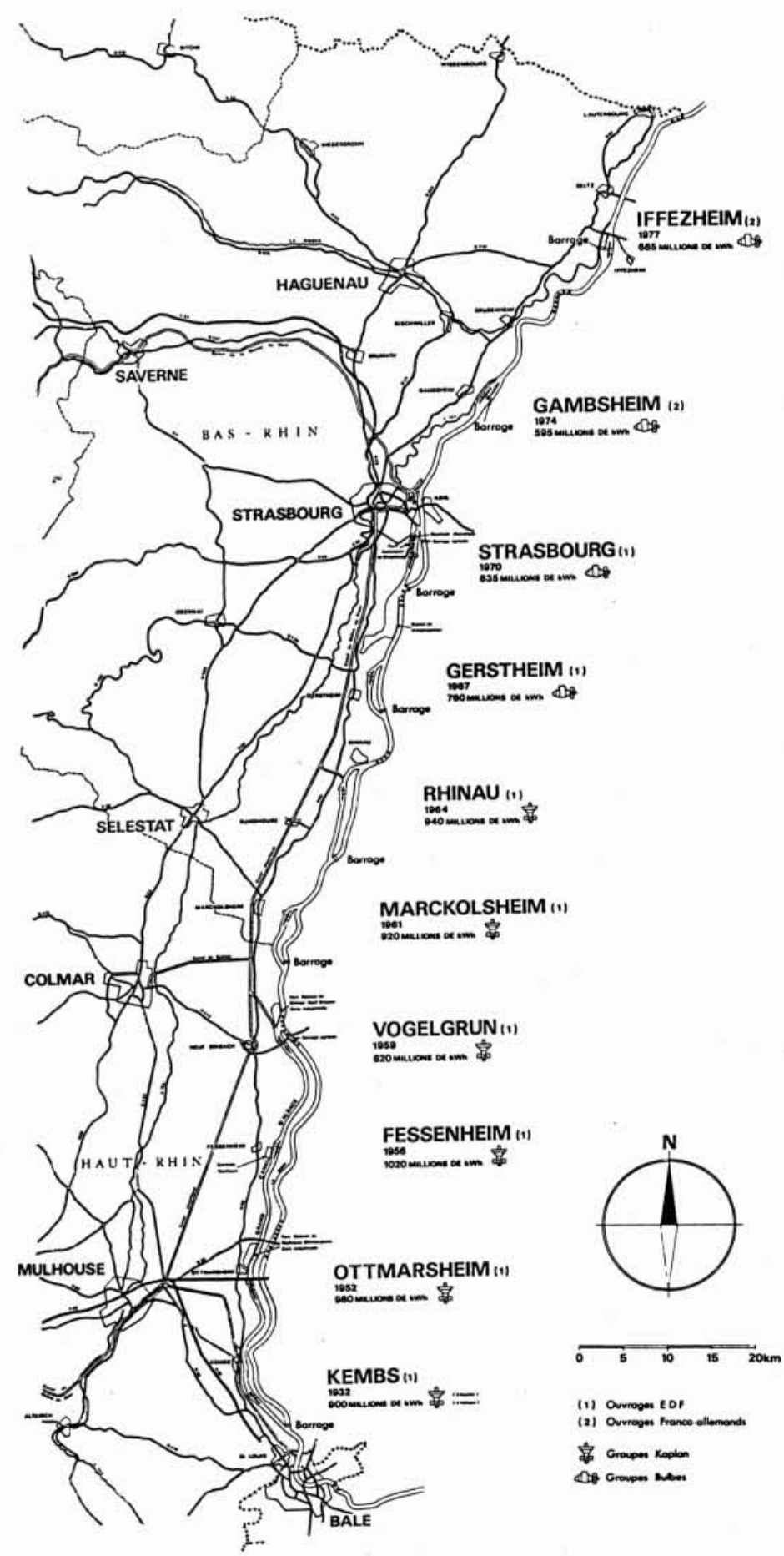

1. 


\section{Remote control of river Rhine plants}

The river Rhine french german portion includes 10 hydroelectric developments built from 1932 to 1977. The main exploitation constraints are those linked to navigation conditions. The present operating system enables to take account of those constraints and it ensures daily modulations of the powerplants production. The "Poste de Sécurité du Rhin", set up in Kembs since 1976, is the chief organ of this control system. It will be changed for a "Poste hydraulique de vallee " by 1994.

\section{Les contraintes d'exploitation}

Les principales contraintes d'exploitation de la chaîne d'aménagements sont celles induites par la navigation fluviale et sont de deux types:

- obligation de maintenir le niveau de chaque bief autour d'une certaine cote ;

- obligation de restituer à l'aval de la chaîne le débit précédemment entré à l'amont.

\section{Maintien des niveaux}

A chaque bief correspond un point dit « Point de réglage " (amont barrage ou usine en général) dont le niveau doit être maintenu dans une certaine fourchette autour de la cote dite de « retenue normale». La fourchette autorisée est en général de l'ordre de $+10 \mathrm{~cm} /-60 \mathrm{~cm}$, sauf pour le bief de Kembs où elle est de $+5 \mathrm{~cm} /-10 \mathrm{~cm}$.

\section{Restitution du débit entrant}

Le cours du Rhin devenant libre à l'aval d'Iffezheim, dernière usine de la chaîne, les niveaux de cette portion du fleuve sont directement liés à son débit. Afin de ne pas créer de fluctuations anormales de niveau, le débit restitué à Iffezheim se doit d'être égal au débit entrant par le Rhin et ses affluents. Cette égalité doit être vérifiée à tout instant moyennant différents retards et filtrages prenant en compte la propagation de l'eau.

Cette contrainte peut également se traduire par l'interdiction d'effectuer de stockage autre que celui découlant de l'augmentation de débit (les pertes de charge le long de chaque bief et par conséquent la pente de chaque ligne d'eau dépendant du débit, le volume d'eau stocké dans un bief, dépend du débit le traversant et de sa cote au point de réglage).

\section{La conduite des aménagements}

\section{Principe général}

Afin de respecter les contraintes mentionnées ci-dessus chacun des 10 aménagements est piloté automatiquement suivant le même principe général (cf. fig. 2).
L'ensemble des fonctions des différents automatismes de la chaîne peut être découpé en deux sous-ensembles :

- une fonction "régulation de débit» qui vise à déterminer pour chaque bief une consigne effective de débit sortant à partir de mesures physiques (débits amont, niveaux de chaque bief...);

- une fonction " asservissement des organes " qui pilote chaque organe réglant (vannages de groupes hydroélectriques, vannes déchargeur ou vannes des barrages mobiles) en cherchant à respecter la consigne de débit sortant.

\section{Détermination des consignes de débit et de niveau}

La détermination des consignes de débit et de niveau de chaque bief doit être faite de telle sorte que les contraintes d'exploitation de la chaîne d'aménagements soient toujours respectées.

Pour pouvoir maintenir constamment le niveau réel de chaque bief à l'intérieur de sa plage de variation autorisée, chaque niveau de consigne doit toujours se situer dans une plage de variation plus petite et incluse dans la précédente (en général la consigne de niveau peut varier dans la plage de $0 /-50 \mathrm{~cm}$ par rapport à la retenue normale pour une plage de niveaux réels autorisés de $+10 /-60 \mathrm{~cm}$ ). La marge de réglage existant entre ces deux plages de réglage est destinée à couvrir les imprécisions inévitables de la conduite qui conduisent à des écarts entre les valeurs réelles et de consigne du niveau de chaque bief. Ces écarts ont cependant une moyenne temporelle nulle et se situent toujours à l'intérieur de la marge de réglage grâce à l'action du correcteur de niveau de chaque bief.

Pour pouvoir respecter la contrainte de restitution du débit entrant, la consigne de débit d'Iffezheim, dernier aménagement de la chaîne, doit être déterminée aussi proche que possible de la somme retardée et filtrée des différents débits entrants dans la chaîne. Pour que les actions des correcteurs de niveau ne viennent pas perturber le débit sortant de ce dernier aménagement, il est nécessaire que les consignes de niveau des différents biefs ne varient que de façon coordonnée, sans conduire à une variation du volume global de l'ensemble des biefs qui pourrait perturber le débit sortant de la chaîne (en fait il faut que la somme pour l'ensemble des 10 biefs des produits écart de niveau par surface apparente soit constante afin de ne pas effectuer de stockage). 
En pratiquant de cette façon, les fonctions " correcteur de niveau" de chaque bief ne sont sollicitées que pour corriger des erreurs de $2^{\mathrm{e}}$ ordre, l'essentiel de la conduite étant assuré par la détermination des différentes consignes de débit. Celles-ci doivent être déterminées de telle sorte qu'elles se rapprochent le plus possible du débit nécessité par le suivi de la consigne de niveau, compte tenu de celui entrant dans le bief.

\section{Réglage du débit par le barrage mobile}

Lorsque le débit devant transiter par un bief est supérieur à celui dit "de saturation" de son usine $\left(1400 \mathrm{~m}^{3} / \mathrm{s}\right.$ en général), le débit de celle-ci est maintenu constant à sa valeur de saturation et le réglage du plan d'eau est effectué par les vannes du barrage. Dans ce mode de fonctionnement le principe général de régulation défini précédemment est appliqué par des automatismes spécifiques implantés sur le site même de chaque barrage. Ceux-ci pilotent alors directement les vannes (partie " asservissement des organes ») et déterminent eux-mêmes les consignes de niveau (en général constantes) et le débit en fonction des mesures du niveau du bief et du débit de l'aménagement amont (partie " régulation de débit »).

\section{Réglage du débit par l'usine}

Lorsque l'usine n'est pas saturée, les vannes du barrage mobile sont fermées et le plan d'eau est régulé par les groupes et vannes déchargeurs de l'usine. Dans ce mode de fonctionnement le principe général de régulation est appliqué par des automatismes implantés d'une part dans chaque usine (partie "asservissement des organes" et fonction "correcteur de niveau " de la partie "régulation de débit ») et d'autre part à Kembs au "poste de sécurité du Rhin" (P.S.R.) (fonction "détermination des consignes" de la partie "régulation de débit »).

\section{Remarques :}

- Puisqu'ils n'ont pas de barrage propre, seul ce mode de réglage est appliqué aux aménagements d'Ottmarsheim, Fessenheim et Vogelgrun)

- Même lors de ce mode de réglage les automatismes du barrage fonctionnent et surveillent le niveau du bief. En cas de dépassement de plus de $30 \mathrm{~cm}$ par rapport à la consigne, les vannes du barrage peuvent s'ouvrir pour réguler le bief à cette valeur (sur défaut non détecté des automatismes de l'usine par exemple).

\section{Le poste de sécurité du Rhin (P.S.R.)}

\section{Présentation du P.S.R.}

Le P.S.R. a été mis en service à partir de 1976 dans les locaux de l'usine de Kembs. Il était à l'origine constitué essentiellement d'un calculateur T 2000/20 de SEMS, doublé en 1982 par un autre de même type afin d'assurer la redondance du système. Un des deux calculateurs est désormais actif tandis que l'autre le surveille et peut devenir actif à sa place en cas de besoin.

Les calculateurs sont reliés aux automatismes (en général de type électromécanique) de chaque usine par l'intermédiaire d'équipements ETC 50 de CETT et de lignes téléphoniques permanentes.

Les principales fonctions du P.S.R. sont :

- la téléconduite des usines du Rhin ;

- la télésurveillance des aménagements du Rhin.

\section{La téléconduite des usines du Rhin}

Suivant les principes de conduite décrits précédemment, le P.S.R. a en charge la détermination des consignes de

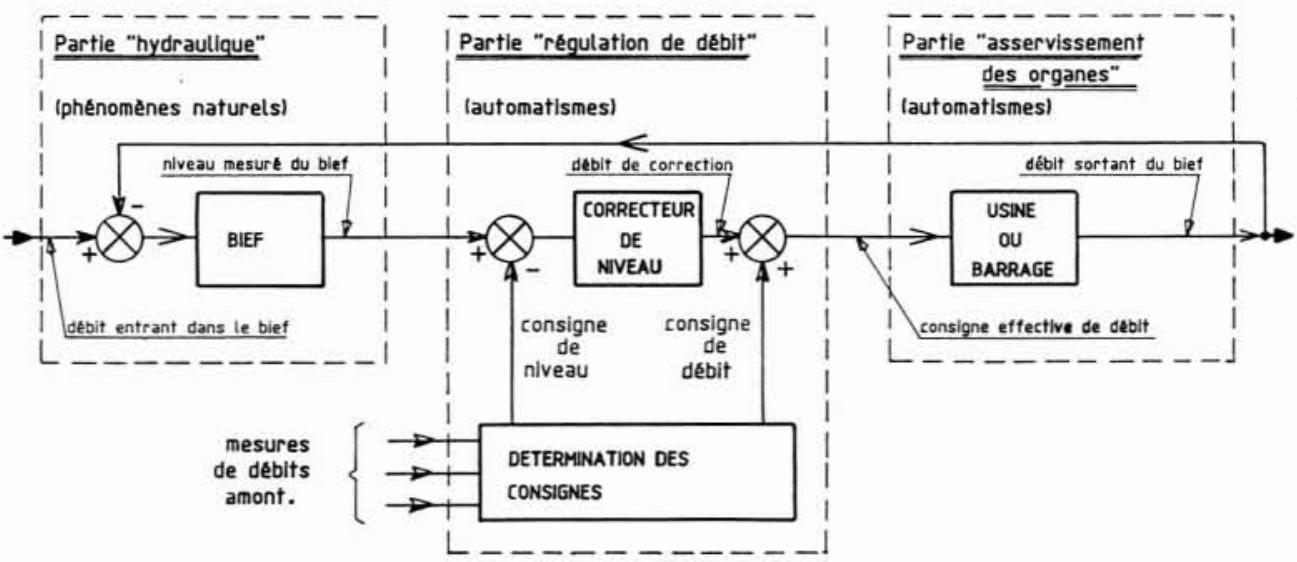

2. Principe général de la conduite. 


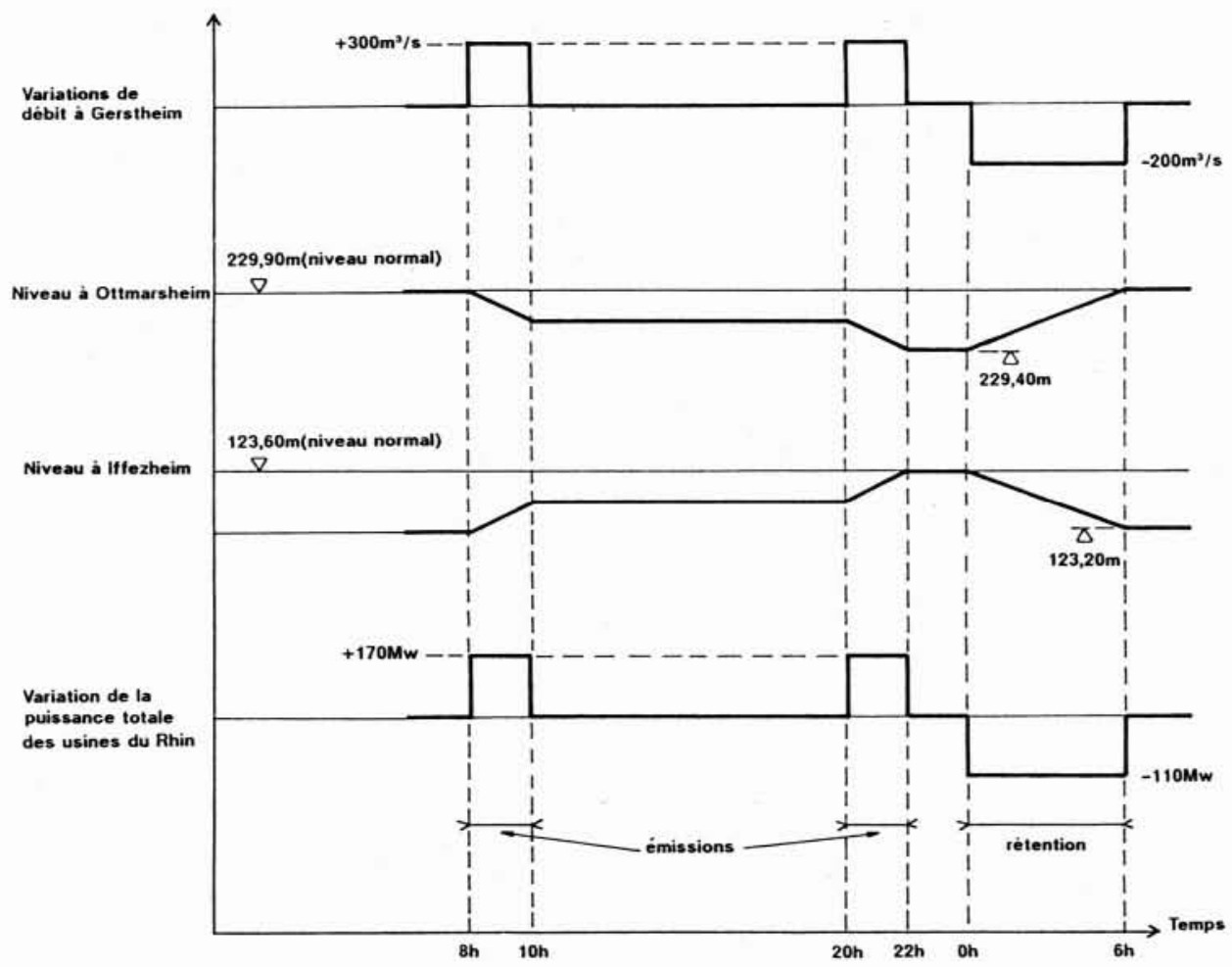

3. Exemple de diagrammes d'éclusée.

niveau et de débit de chaque aménagement en deçà de son débit de saturation.

La consigne de débit est constituée de deux termes : un terme "fil de l'eau " et un terme "éclusées " déterminés chacun par une partie correspondante de la conduite. La consigne de niveau est quand à elle intégralement calculée par la partie "éclusées" de la conduite.

La conduite au fil de l'eau. Le terme "fil de l'eau» de la consigne de débit envoyée à chaque usine est déterminé comme si le niveau de chaque bief devait rester constant en respectant la contrainte de restitution du débit entrant.

Pour réaliser cette fonction le P.S.R. mesure le débit entrant par le Rhin ou ses affluents par l'intermédiaire d'une mesure de niveau (les points de mesure de ces niveaux étant dans des portions où les différents cours d'eau sont à écoulement libre, il existe des correspondances qui permettent de déterminer leur débit à partir de la mesure de leur niveau). Les variations détectées de débit entrant sont alors successivement répercutées avec un retard croissant sur les consignes de débit des usines aval. Une variation de débit du Rhin à Bâle sera ainsi immédiatement répercutée sur la consigne de débit de l'usine de Kembs, après 10 minutes sur celle d'Ottmarsheim, 29 minutes sur celle de Fessenheim et ainsi de suite jusqu'à Iffezheim où le retard atteint 2 heures.

La conduite en éclusées (cf. fig. 3). Les éclusées sont des opérations dont le but est de moduler la puissance de l'ensemble des usines afin de l'adapter, dans une certaine mesure, à celle souhaitable pour le réseau électrique Français. Ce type de conduite se superpose à celui du fil de l'eau et est à l'origine des consignes de niveau de chaque bief ainsi que des termes "éclusées " des consignes de débit.

Par rapport à celle découlant du fil de l'eau, les éclusées permettent d'augmenter la puissance totale des usines pendant les périodes d'"émission" (correspondant aux heures chargées du réseau) et de la diminuer pendant les périodes de "rétention " (correspondant aux heures creuses).

Une émission consiste en un déstockage d'eau de certains biefs amont dits "émetteurs" (biefs de Ottmarsheim à Marckolsheim en général), un transfert par des biefs intermédiaires dits "transmetteurs" (Rhinau à Gerstheim en général) et un stockage dans des biefs aval dits « récepteurs » (Strasbourg, Gambsheim et Iffezheim en général). Une rétention consiste en la manœuvre inverse.

La plage de variation de puissance peut aller jusqu'à $+200 \mathrm{MW}$ pendant 4 heures et $-140 \mathrm{MW}$ pendant 6 heures suivant les conditions de débits.

Les calculateurs du P.S.R. ont en mémoire les caractérístiques des émissions et des rétentions des 24 heures à venir (heure de début, durée, amplitude de variation...). Ces caractéristiques sont reconductibles d'un jour sur l'autre ou modifiables par le chef de quart du P.S.R. suivant les conditions de débit du moment et les besoins du réseau transmis téléphoniquement par le dispatching régional de Nancy.

\section{LA HOUILLE BLANCHE/N²-1991}


Au début de chaque phase active d'éclusée (émission ou rétention) le P.S.R. modifie le terme "éclusées " des consignes de débit de chaque usine concernée (au début d'une émission par exemple chacun passe de 0 à + DQ, DQ étant une valeur propre à chaque bief et pouvant aller jusqu'à $300 \mathrm{~m}^{3} / \mathrm{s}$ ). Pendant les phases actives le P.S.R. effectue le suivi des consignes de niveau suivant des rampes déterminées en fonction des différences entre les termes « éclusées" des consignes de débit des usines amont et aval (pendant une émission par exemple, la pente de variation de la consigne de niveau est négative pour les biefs émetteurs, nulle pour les transmetteurs et positive pour les récepteurs).

Remarque : afin de respecter les contraintes d'exploitation, le bief de Kembs ne participe jamais aux éclusées (à cause de la tolérance de $+5 /-10 \mathrm{~cm}$ sur son niveau) et le terme "éclusées" de la consigne de débit de l'usine d'Iffezheim est en général nul (pour ne pas créer de fluctuations à l'aval).

\section{La télésurveillance des aménagements du Rhin}

Un certain nombre d'informations permettant une surveillance globale des 10 aménagements est disponible au P.S.R. Ces informations peuvent être relatives à un organe d'une usine (état d'un groupe hydroélectrique ou d'une vanne par exemple), à l'ensemble d'un aménagement (mesure de niveaux ou état du barrage par exemple), ou à l'ensemble des aménagements (niveaux du Rhin et des affluents par exemple). Les calculateurs du P.S.R. peuvent, suivant l'état ou la valeur de chacune de ces informations, élaborer des différentes alarmes à destination de l'opérateur, seule personne effectuant une surveillance permanente de l'ensemble de la chaîne.

Remarque: les automatismes de chaque aménagement détectent également des alarmes à destination du personnel local qui peut alors intervenir directement sur le site.

\section{L'exploitation du P.S.R.}

L'exploitation du P.S.R. est effectuée en permanence par un opérateur, le "Chef de Quart du P.S.R. ", par l'intermédiaire d'écrans cathodiques, d'imprimantes et d'enregistreurs graphiques. Il peut, en cas de besoin, envoyer des ordres à destination d'un aménagement ou du P.S.R. à l'aide d'un clavier et d'une platine de commande spécialisée. Ces équipements lui permettent également d'effectuer des opérations non automatiques (modification d'un programme d'éclusées par exemple) ou éventuellement de corriger le fonctionnement du processus automatique (modification d'une consigne de débit par exemple).

\section{Perspectives d'évolution de la téléconduite}

Afin d'encore améliorer les performances de la téléconduite et de faire face à l'obsolescence des différents matériels, le système actuel de conduite sera en partie renouvelé d'ici à 1994.

Le nouveau système est formé d'un Poste Central de Poste Hydraulique de Vallée (PC de P.H.V.) destiné à remplacer le P.S.R. et de 10 Postes Asservis (PA) remplaçant une partie des automatismes de chaque usine.

Bien que ce nouveau système présente des nouveautés importantes, il peut être considéré comme la suite logique de son prédécesseur puisqu'il reprend, en les améliorant les principes de conduite définis précédemment. Outre une meilleure conviviabilité vis-à-vis de ses différents utilisateurs, les améliorations portent essentiellement sur :

- la détermination du débit de consigne de chaque bief par le PC de P.H.V.;

- la détermination des différents débits de correction par chaque PA ;

- la souplesse d'utilisation des éclusées par le dispatching.

Détermination des consignes de débit par le PC de P.H.V.

Alors que le P.S.R. ne prenait en compte que les variations des débits à leur entrée dans la chaîne d'usines, le P.H.V. détermine le terme "fil de l'eau" de chaque débit de consigne à partir de ces mêmes variations mais aussi à partir de celles de la différence "mesure moins consigne" du débit de chaque aménagement amont. Cette nouvelle méthode permet de limiter l'influence des correcteurs de niveau des diffërents biefs sur le débit à Iffezheim (en ne tenant pas compte de la différence "mesure moins consigne » du débit de l'aménagement amont, une perturbation de niveau dans un bief quelconque se transmet à tous les biefs aval en étant à chaque fois amplifiée par l'action des correcteurs de niveau).

Les différents coefficients intervenant dans le calcul de ces consignes sont déterminés de façon optimale à l'aide d'une simulation numérique effectuée au centre de calcul E.D.F. de Clamart. Cette simulation utilise un modèle mathématique représentant les écoulements dans l'ensemble des 10 biefs du Rhin.

\section{Détermination des débits de correction par chaque $P A$}

Alors que la fonction " correcteur de niveau " du système actuel était effectuée dans chaque usine par un correcteur de type Proportionnel-Intégral (PI) réglant un niveau réel, le nouveau système intègre dans chaque PA un correcteur numérique à coefficients variables qui régule un niveau fictif calculé à partir de la mesure de plusieurs niveaux réels. Ce système effectue une régulation du niveau de chaque bief vis-à-vis du terme " fil de l'eau " de la consigne de débit (variations de consignes transmises d'une usine sur l'autre avec un retard) et une régulation du volume d'eau stocké dans le bief vis-à-vis du terme "éclusées " de la consigne de débit (variations de consignes synchrones dans les usines).

Les différents coefficients de ce correcteur sont également déterminés de façon optimale par simulation numérique. 
Amélioration de la souplesse des éclusées vis-à-vis du dispatching

Avec le nouveau système la puissance totale des usines du Rhin pourra être décomposée en deux parties; une « fil de l'eau ", et une " éclusées » liées chacune aux termes correspondants des consignes de débit. Alors qu'il n'aura toujours aucune action sur la première partie, le dispatching pourra utiliser la seconde comme s'il utilisait une Station de Transfert d'Energie par Pompage (STEP) du genre de celles de Revin ou Grand Maison par exemple.

Les performances de cette STEP fictive dépendant des conditions hydrologiques et des disponibilités des différentes machines, le PC de P.H.V. calculera périodiquement puis transmettra au dispatching par une liaison intercalculateurs les caractéristiques du moment de cet aménagement fictif (jusqu'à $+200 \mathrm{MW}$ pendant 4 heures). En retour, le dispatching enverra des diagrammes liés à la puissance souhaitée pour cette STEP. Ces diagrammes seront ensuite transformés en programmes d'éclusées par le PC de P.H.V

\section{Planning de mise en place du nouveau système}

- Juillet 1989: commande auprès de la Société CEGELEC, Division ERE-P de l'ensemble des PA.

- Décembre 1989 : commande auprès de la même Société du PC de P.H.V.

- Avril 1991 : mise en service du premier PA (Kembs).

- Mars 1992: mise en service du PC de P.H.V.

- Août 1993 : mise en service des derniers PA (Gambsheim et Iffezheim).

- 1994 : dépose du P.S.R.

Remarque: l'installation des PA se faisant de façon progressive, les deux systèmes ancien et nouveau devront cohabiter et piloter chacun une partie des aménagements pendant toute la période comprise entre avril 1991 et août 1993.

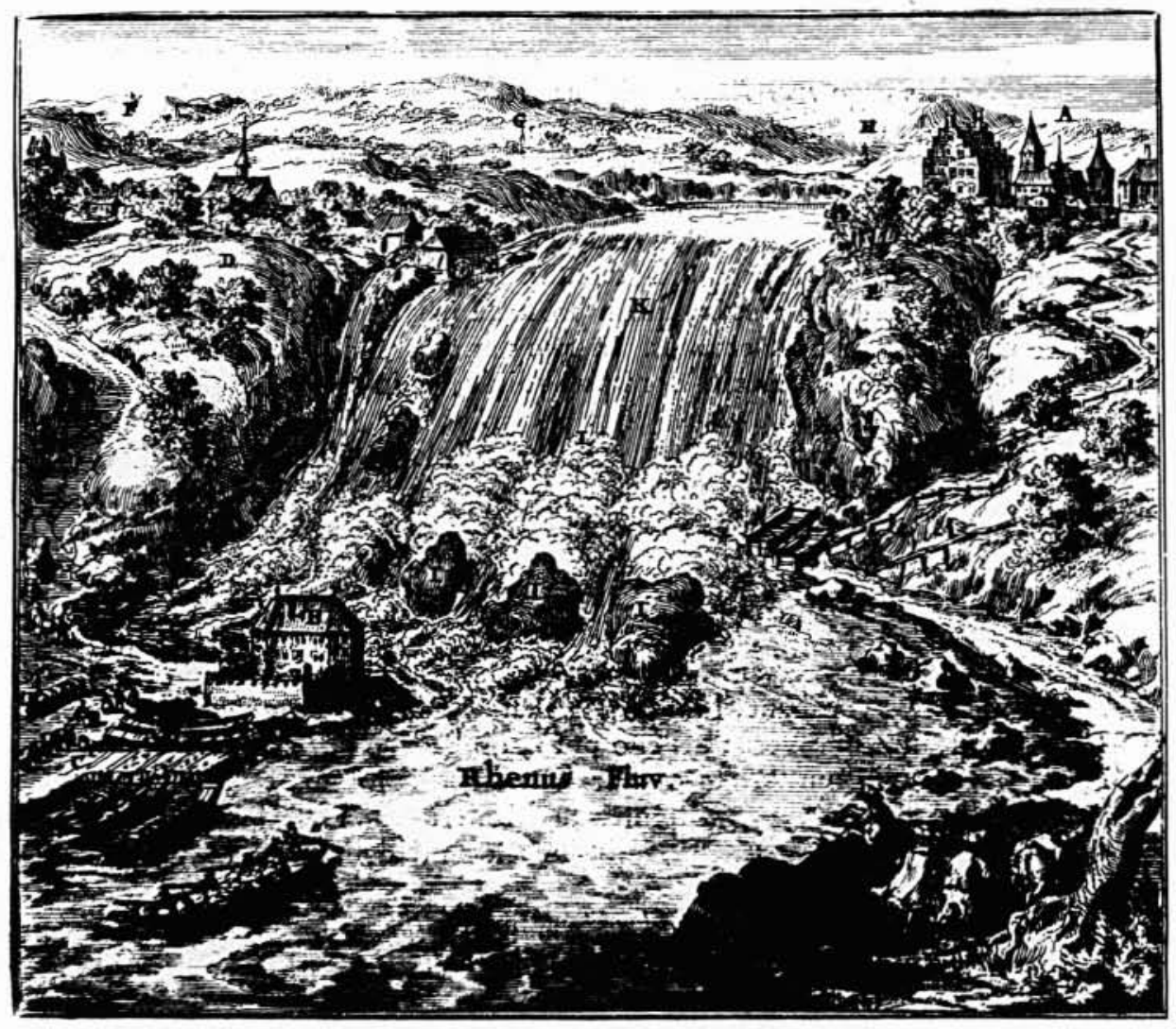

